Dr. Martin deduces in his paper a number of formulæ which lead to very remarkable numerical results.

In Professor White's paper the problem is to reduce the resultant of a binary quadric and $n$-ic to a sum of products of invariants of the "reduced system." Clebsch, and later Gordan, have solved it. They use unnecessary auxiliaries. The reduction is accomplished more speedily by constructing synthetically, according to the method of the theory of forms, an expression involving undetermined coefficients whose values are then found by the differential equation of apolarity applied to that covariant which becomes an exact power of the common factor of the two quantities in case their resultant vanishes. Incidentally the connection between apolarity and the semicombinant property is illustrated.

Thomas S. Fiske.

\title{
ON THE CONNECTION BETWEEN BINARY QUARTICS AND ELLIPTIC FUNCTIONS.*
}

BY PROFESSOR E. STUDY.

AFTER the treatment which the subject has received in the recent work of Professors Harkness and Morley, it may be supposed that American mathematicians are quite familiar with the utilization of the theory of invariants in the theory of elliptic functions. We shall confine ourselves to the simplest case, where the theory of a binary quartic is concerned. We intend to show how a certain group of rational and irrational covariants of a binary quartic can be expressed as one-valued functions of one or two parameters, thus filling up a number of lacunae contained in former presentations of the subject.

After having explained the system of notation we are to apply, we proceed, partly following Cayley, to define a systern of irrational covariants. These being known, we compare these quantics with the elliptic $\theta$-functions; and thus we will be enabled to express the connection in question in very simple terms.

Of course we must suppress here not only the proofs, but also quite a number of details; a full exposition will be published shortly in the American Journal of Mathematics.

\section{Notation.}

Denoting the quartic $f(x)$ symbolically by $f=(a x)^{4}$ $=\left(a_{1} x_{2}-a_{2} x_{1}\right)^{4}$, the following forms constitute, as is well known, what is termed the complete system of $f$ :

* This puper, which Professor Study kindly transmitted for presen tation at the Brooklyn meeting of the Ambrican Mathematical So. CIETY, arrived too late for that purpose. 


$$
\begin{aligned}
& \begin{array}{l|l}
f=(a x)^{4} \\
h=\frac{1}{2}(a b)^{2}(a x)^{2}(b x)^{2}=(h x)^{4}
\end{array} \\
& t=2(a h)(a x)^{9}(h x)^{3}=(t x)^{6} \quad T \quad \text { (Notation applied by } \\
& g_{2}=\frac{1}{2}(a b)^{4} \\
& g_{\mathrm{s}}=\frac{1}{3}(a h)^{4}=\frac{1}{6}(a b)^{2}(a c)^{2}(b c)^{2}\left|\frac{j}{6}\right| \\
& G=\frac{1}{16}\left(g_{2}^{3}-27 g_{3}^{2}\right)
\end{aligned}
$$

is the discriminant.

2. Decomposition of $f$ into its linear factors.

In order to decompose $f$ into linear factors, which are to be irrational covariants of $f$, we have first to decompose the Jacobian $t$ into its three conjugate quadratic factors by means of the solution of the cubic equation

$$
4 x^{3}-g_{2} x-y_{2}=0 \text {. }
$$

We denote the roots by $e_{\lambda}, e_{\mu}, e_{\nu} . \quad h+e_{\lambda} f$ being a perfect square, we are enabled to define a set of three quadratic forms $l, m, n$ by means of the formulæ

$$
l=(l x)^{2}=\sqrt{\frac{\hbar+e_{\lambda} f}{\left(e_{\mu}-e_{\lambda}\right)\left(e_{\nu}-e_{\lambda}\right)}}, \text { etc., }
$$

restricting the meaning of the radicals by the further supposition

$$
(m n)(m x)(n x)=-(l x)^{2}, \text { etc. }
$$

Then we have

$$
t=-2 \sqrt{G} \cdot(l x)^{2} \cdot(m x)^{2} \cdot(n x)^{2} .
$$

Writing now

$$
f=4\left(r_{0} x\right) \cdot\left(r_{\lambda} x\right) \cdot\left(r_{\mu} x\right) \cdot\left(r_{\nu} x\right),
$$

we may define the linear forms $\left(r_{\lambda} x\right)$, irrational covariants of $f$, by means of the formulæ

$$
\left\{\begin{array}{l}
2\left(r_{0} x\right)^{2}=-\sqrt{e_{\mu}-e_{\nu}} \cdot l-\sqrt{e_{\nu}-e_{\lambda}} \cdot m-\sqrt{e_{\lambda}-e_{\mu}} \cdot n, \\
2\left(r_{\lambda} x\right)^{2}=-\sqrt{e_{\mu}-e_{\nu}} \cdot l+\sqrt{e_{\nu}-e_{\lambda}} \cdot m+\sqrt{e_{\lambda}-e_{\mu}} \cdot n, \\
2\left(r_{\mu} x\right)^{2}=\sqrt{e_{\mu}-e_{\nu}} \cdot l-\sqrt{e_{\nu}-e_{\lambda}} \cdot m+\sqrt{e_{\lambda}-e_{\mu}} \cdot n, \\
2\left(r_{\nu} x\right)^{2}=\sqrt{e_{\mu}-e_{\nu}} \cdot l+\sqrt{e_{\nu}-e_{\lambda}} \cdot m-\sqrt{e_{\lambda}-e_{\mu}} \cdot n,
\end{array}\right.
$$

the expressions on the right being again perfect squares. 
By these formulæ, which are due to Cayley, the forms $\left(r_{\lambda} x\right)$ are not yet fully determined. In order to restrict their ambiguity as much as possible, we have to add some further conditions. These are furnished by a remarkable fact, which seems to have escaped notice hitherto: The bilinear invariants $\left(r_{i} r_{k}\right)$ belong to the domain of rationality defined by the quantities $\sqrt{e_{\mu}-e_{\nu}}$, etc.

As a close examination shows, it is possible to restrict the values of the quantities $\left(r_{k} x\right)$ by the further suppositions

$$
\left\{\begin{array}{l}
\left(r_{\mu} r_{\nu}\right)=-\left(r_{o} r_{\lambda}\right)=\sqrt{e_{\mu}-e_{\nu}} \\
\left(r_{\nu} r_{\lambda}\right)=-\left(r_{o} r_{\mu}\right)=\sqrt{e_{\nu}-e_{\lambda}} \\
\left(r_{\lambda} r_{\mu}\right)=-\left(r_{0} r_{\nu}\right)=\sqrt{e_{\lambda}-e_{\mu}}
\end{array}\right.
$$

'The signs of the square roots on the right being chosen, and $l, m, n$ being known, the linear factors $\left(r_{\kappa} x\right)$ of $f$ are completely determined, excepting a change of sign of the whole set, which remains still possible.

\section{Introduction of the $\theta$-functions.}

Now an examination of the relations among the linear forms $\left(r_{\kappa} x\right)$ furnishes the very important result, that these relations are exactly identical with the relations among the squares of the four elliptic $\theta$-functions-provided that the definition of the $\theta$-functions which has been given by the author in his paper (American Journal, vol. 16, No. 2) is accepted.

Identifying, therefore, the quantities $\sqrt[4]{e_{\mu}-e_{\nu}}$, taken from the theory of the quartic, with the quantities $\sqrt[4]{e_{\mu}-e_{\nu}}=$ $\theta_{\lambda}(0)$, occurring in the theory of elliptic functions, we are enabled to explain a set of square roots by means of the formulæ

$$
\begin{gathered}
\sqrt{\left(r_{\kappa} x\right)}=\theta_{\kappa} u(\kappa=o, \lambda, \mu, v), \\
\sqrt{f}=-2 \theta u \theta_{\lambda} u \theta_{\mu} u \theta_{\nu} u .
\end{gathered}
$$

These formulæ contain the clue to the whole theory in question. It is easy now to derive all known results and a large number of new ones; and this is done by means of actual calculations, without any help of the so-called method of indeterminate coefficients.

We limit ourselves to communicating some of the most interesting results. first

Passing from the single to the double argument, we obtain 


$$
\left\{\begin{aligned}
\sqrt{f^{\prime}} & =-\theta^{\prime} \cdot \theta(2 u), \\
l & =-\theta_{\lambda} \cdot \theta_{\lambda}(2 u), \\
m & =-\theta_{\mu} \cdot \theta_{\mu}(2 u), \\
n & =-\theta_{\nu} \cdot \theta_{\nu}(2 u),
\end{aligned}\right.
$$

where $\theta_{\lambda}$ and $\theta^{\prime}$ mean $\theta_{\lambda}(0)$ and $\theta^{\prime}(0)=\theta_{\lambda} \theta_{\mu} \theta_{\nu}$; and considering two pairs of variables $x, u$ and $y, v$ at the same time, we have

$$
\left\{\begin{aligned}
(x y) & =-\theta(u+v) \theta(u-v), \\
(l x)(l y) & =-\theta_{\lambda}(u+v) \theta_{\lambda}(u-v), \\
(m x)(m y) & =-\theta_{\mu}(u+v) \theta_{\mu}(u-v), \\
(n x)(n y) & =-\theta_{\nu}(u+v) \theta_{\nu}(u-v),
\end{aligned}\right.
$$

or expressions of the identical covariant $(x y)=x_{1} y_{2}-x_{2} y_{1}$ and of the polars of the quadratic forms $(l x)^{2},(m x)^{2},(n x)^{2}$ in terms of $u, v$.

These formulæ, especially the equations (4), are not only worth noticing for their symmetry and elegance, but they are also very useful; they are the true central point of our theory. By the formulæ (4) the general addition theorems of Jacobi and Weierstrass are brought into close connection with the properties of the four bilinear forms on the left. It is remarkable that these very forms bear a simple relation still to another theory: the composition of our forms is exactly identical with the multiplication of the four quaternion-units.

\section{The elliptic funetions øu, $\wp^{\prime} u$.}

By means of the preceding formulæ Weierstrass' functions $\wp u, \wp^{\prime} u$ are easily expressed in terms of covariants of $f$.

Supposing $\omega_{0}=0$, and denoting the periods corresponding to $\theta_{\lambda}, \theta_{\mu}, \theta_{\nu}$, as usual by $2 \omega_{\lambda}, 2 \omega_{\mu}, 2 \omega_{\nu}$, we have first a group of irrational formulæ:

$$
(5)\left\{\begin{aligned}
\wp\left(u+\omega_{\kappa}\right) & =\frac{\left(t r_{\kappa}\right)^{6}(t x)}{2 \sqrt[4]{G} \cdot\left(a r_{\kappa}\right)^{3}(a x)}=\frac{\left(t r_{\kappa}\right)^{5}(t x)}{2 \sqrt{G} \cdot\left(r_{\kappa} x\right)}, \\
\wp^{\prime}\left(u+\omega_{\kappa}\right) & =\sqrt[4]{G} \cdot \frac{\sqrt{f}}{\left(r_{0} x\right)^{2}}, \\
\wp^{\prime \prime}\left(u+\omega_{\kappa}\right) & =\frac{3\left(t r_{\kappa}\right)^{4}(t x)^{2}}{\sqrt[4]{G} \cdot\left(r_{\kappa} x\right)^{2}}, \quad \text { etc. } \quad\left(\kappa=0, \lambda, \mu, v_{\bullet}\right)
\end{aligned}\right.
$$


For the elliptic functions of the double argument, we obtain simpler expressions, namely, Hermite's famous formulæ,

$$
\left\{\begin{array}{l}
\wp(2 u)=-\frac{h}{f}, \\
\wp^{\prime}(2 u)=\frac{t}{f \sqrt{f}}, \text { etc. }
\end{array}\right.
$$

Considering again two pairs of variables, we find further the remarkable formulæ

$$
\left({ }^{7}\right)\left\{\begin{array}{l}
\wp(u \pm v)=\frac{(a x)^{2}(a y)^{2} \mp \sqrt{(a x)^{4}} \sqrt{(a y)^{4}}}{2(x y)^{2}} \\
\wp^{\prime}(u \pm v)=-\frac{(a y)^{3}(a x) \cdot \sqrt{(a x)^{4}} \mp(a x)^{8}(a y) \sqrt{(a y)^{4}}}{(x y)^{3}}
\end{array}\right.
$$

the content of which is partly due to Weierstrass.

It follows now that all covariants of the quartic f containing the two sets of variables $\left(x_{1}, x_{2}\right)$ and $\left(y_{1}, y_{2}\right)$ in the degree zero are rational functions of $\wp(u+v), \wp(u-v), \wp^{\prime}(u+v)$, $\wp^{\prime}(u-v)$, and vice versa-provided we include in the co. variants of $f$ the two irrationalities $\sqrt{f_{x}}$ and $\sqrt{f_{y}}$.

In particular, when a power of $(x y)$ is the sole denominator, the covariant in question is an integral function of the four elliptic functions mentioned.

A few simple examples will show the significance of the last theorem. We have

$$
\begin{aligned}
& \wp(u+v) \cdot \wp(u-v)+\frac{1}{12} g_{2}=-\frac{(h x)^{2}(h y)^{2}}{(x y)^{2}}, \\
& \wp^{\prime}(u+v) \cdot \wp^{\prime}(u-v)=-2 \frac{(t x)^{3}(t y)^{3}}{(x y)^{3}}, \\
& \wp(u \pm v) \cdot \wp^{\prime}(u \mp v)=\frac{(h y)^{3}(h x) \cdot \sqrt{(a x)^{4}} \pm(h x)^{3}(h y) \sqrt{(a y)^{4}}}{(x y)^{3}}, \\
& \frac{1}{3} \wp^{\prime}(u \pm v) \cdot \wp^{\prime \prime}(u \pm v)=\frac{(t y)^{4}(t x)^{2} \cdot \sqrt{(a x)^{4}} \pm(t x)^{4}(t y)^{2} \cdot \sqrt{(a y)^{4}}}{(x y)^{4}}, \\
& \text { etc. }
\end{aligned}
$$

